\title{
Limb Ischemic Preconditioning Reduces Heart Injury after Severe Burn Injury in Rats
}

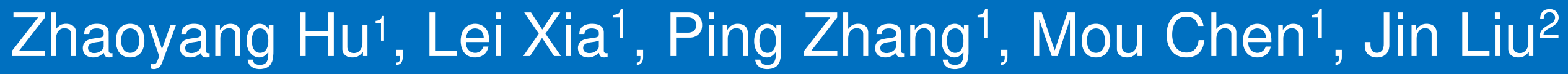

${ }^{1}$ Laboratory of Anesthesiology \& Critical Care Medicine, Translational Neuroscience Center, West China Hospital, Sichuan University, Chengdu,China

${ }^{2}$ Department of Anesthesiology, West China Hospital, Sichuan University, Chengdu,China

\section{Background}

Post-burn cardiac shock causes myocardial damage and cardiac dysfunction at the early stage of severe burns. We showed that remote ischemic preconditioning (RIPC) protects heart against myocardial I/R injury. However, little is known regarding the effect of RIPC on heart after severe burn.

\section{Initial aim of the study}

We proposed the existence of RIPC-induced cardioprotection in severely burned rats and further investigate the underlying mechanisms.

\section{Remote ischemic preconditioning}

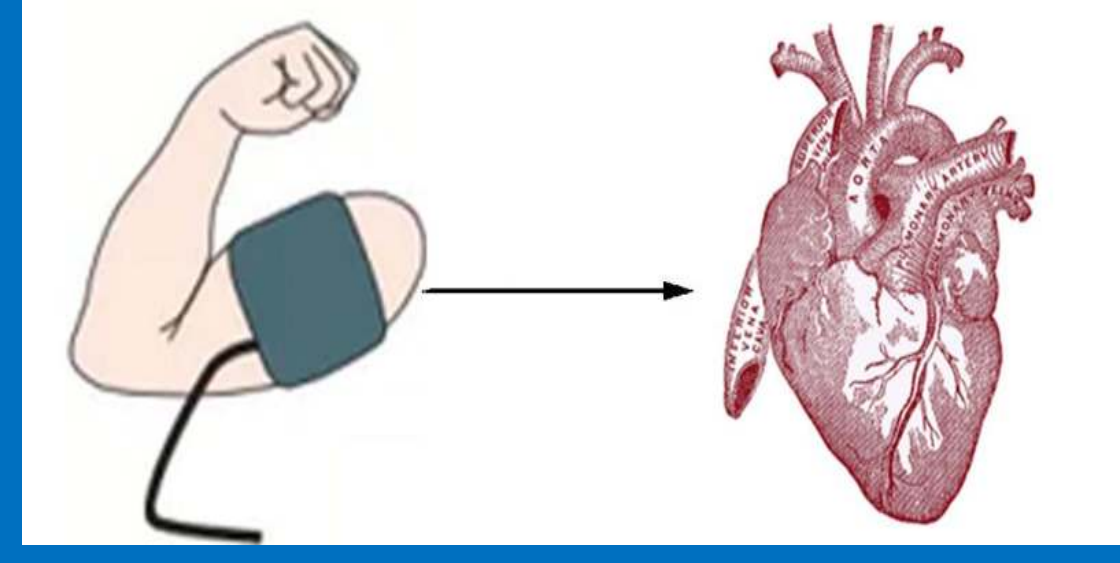

\section{Methods}

Male Sprague Dawley rats were assigned to sham-

burn,control(CON), limb

ischemic preconditioning group (RIPC), each group, $n=8$.

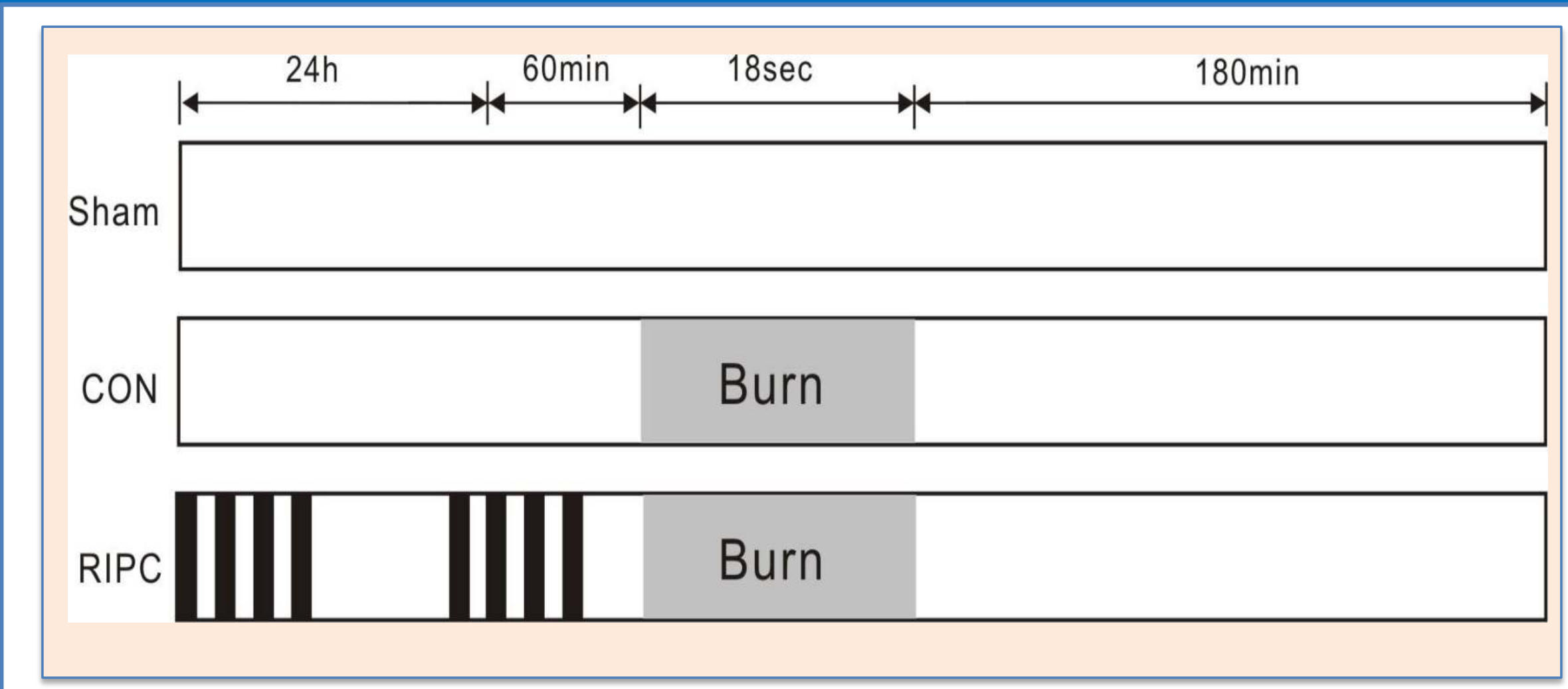

Rats were inflicted with $30 \%$ total body surface area full-thickness burn in a $97^{\circ} \mathrm{C}$ water bath for $18 \mathrm{~s}$ except for the sham rats. Immediately after burn injury, all rats were resuscitated with Ringer's lactate solution (4.0ml $/ \mathrm{kg} / 1 \%$ TBSA,i.p.). For RIPC intervention, rats received four cycles of $5 \mathrm{~min}$ of limb ischemia with 5 min reperfusions at 24 hours and 1 hour before burn injury. Hemodynamics were measured. 3 hours post-burn, hearts were taken for apoptosis measurements and westernblotting analysis. Myocardial mitochondria and cytosol were isolated to determine mitochondrial energy metabolism, cytochrome $c$ release and mitochondrial membrane potential $(\Delta \Psi \mathrm{m})$. Blood samples were taken for serum levels of lactate dehydrogenase (LDH) and creatine kinase-MB (CK-MB) determination.

\section{Results}

RIPC restored cardiac function, increased $\mathrm{Bcl}-2$ expression, decreasing Bax and Caspase-3 expression and enhancing Bcl-2/Bax ratios. RIPC preserved post-burn cardiac mitochondrial function by recovering ATP concentrations and decreasing AMP/ATP ratio ( $\mathrm{P}<0.05$ vs. CON). RIPC upregulated $\Delta \Psi \mathrm{m}$ from $11.2 \pm 2$ in the $C O N$ to $17.2 \pm 1.1 \mu \mathrm{g} / \mathrm{mL}(P<0.05)$. RIPC caused a $40 \%$ lower of cytochrome $c$, than rats in CON $(P<0.01)$. Serum levels of cardiac enzyme leakage were reduced in RIPC group.

RISK pathway (Akt, ERK1/2, and GSK3 $\beta$ ) phosphorylation was enhanced in RIPC-treated hearts compared to non-treated control groups $(P<0.05)$, highlighting the crucial role of the RISK pathway in RIPC-induced heart protection after severe burn injury.

\section{Summary}

Limb ischemic conditioning effectively improves cardiac function, ameliorates myocardial damage and preserves mitochondrial function after severe burn injury. The underlying mechanism may involve RISK signaling pathways. 